\title{
On the finite horizon Nash equilibrium solution in the differential game approach to formation control
}

\author{
JOND Hossein Barghi* and NABIYEV Vasif \\ Department of Computer Engineering, Karadeniz Technical University, Trabzon 61080, Turkey
}

\begin{abstract}
The solvability of the coupled Riccati differential equations appearing in the differential game approach to the formation control problem is vital to the finite horizon Nash equilibrium solution. These equations (if solvable) can be solved numerically by using the terminal value and the backward iteration. To investigate the solvability and solution of these equations the formation control problem as the differential game is replaced by a discrete-time dynamic game. The main contributions of this paper are as follows. First, the existence of Nash equilibrium controls for the discretetime formation control problem is shown. Second, a backward iteration approximate solution to the coupled Riccati differential equations in the continuous-time differential game is developed. An illustrative example is given to justify the models and solution.
\end{abstract}

Keywords: formation control, differential game, dynamic game, Nash equilibrium, coupled Riccati equations.

DOI: $10.21629 /$ JSEE.2019.06.17

\section{Introduction}

Formation control of multi-agent systems is a frontier research topic of control science and has found applications in diverse areas, such as mobile robots $[1,2]$, satellite control [3], unmanned surface vessels (USVs) [4,5], and unmanned aerial vehicles (UAVs) [6,7]. The formation control objective is to design a controller that drives all agents to the desired formation in the state space. To maintain a formation, agents need to exchange information such as relative positions and velocities. Several formation control approaches are introduced, such as behavior-based approach [8], virtual-structure approach [9], leader-follower approach [10], consensus [11], optimal control and differential game [12]. The behavior-based approach is easy to implement but lacks theoretic analysis, since there are no rigorous models of team behaviors. The virtual-structure approach tends to be rather complex in implementation, but the robustness of the formation against uncertainties is achieved [13]. The leader-follower approach is simple and

Manuscript received August 01, 2018.

*Corresponding author. computationally efficient, but the formation can become disjoint if no feedbacks from the leader(s) are given to the followers, and it suffers from poor uncertainties rejection. The optimal control approach is supported by rigorous theories, such as the minimum principle and Hamilton-JacobiBellman equation, but it assumes the agents' future maneuver strategies are defined, while the differential game approach instead takes into consideration their maneuver capabilities [14]. The formation control problem becomes more challenging when the physical limitations for dynamical systems or the presence of obstacles in their workspace need to be addressed. For practical control constrained control laws are needed to be designed. Dynamic positioning vessels in the presence of input amplitude and rate saturations constraints were investigated in [15], and the obstacle avoidance based control law was proposed in [16].

The differential game approach to formation control of mobile robots was introduced in [12]. In this approach, each robot is viewed as a player of a differential game, in which it can select its neighbors; therefore distributed controllers can be implemented. The communication network is modeled as a graph with directed edges corresponding to the flow of information within the system. Distributed formation control of UAVs using the differential game approach was studied in [17]. Each UAV minimizes its terminal formation errors and terminal velocity differences to other UAVs according to the communication graph. In [18], the collision avoidance formation control problem was approached using the differential game. An approximate solution to the game was proposed using a set of matrices satisfying the coupled algebraic Riccati equations (AREs).

The formation control problem can be modeled as a non-cooperative linear quadratic (LQ) differential game. Under the framework of this game, a set of coupled Riccati differential equations (CRDEs) has to be solved. In general, the solution to these equations can often be in- 
tractable or maybe not exist (see [19] for a detailed study of such equations). CRDEs in closed-loop differential games, zero-sum differential games, and infinite-time horizon differential games were studied in [20], [21] and [22,23], respectively. CRDEs appearing in the non-cooperative nonzero sum LQ differential game with the open-loop information structure on a finite-time horizon were investigated in $[24,25]$. An explicit solution of these equations in terms of a solution of the associated ARE was given in [24] in the case of invertibility of an introduced matrix. In [25], the precise integration method from the uncoupled Riccati differential equations in the LQ optimal control problem was expanded to solve the CRDEs in the non-cooperative LQ differential game. This method is applicable if the solutions to the CRDEs exist.

In this paper, the leaderless formation control problem for a multi-robot system, in which all robots have the same role, is formulated as an LQ differential game. Therefore, a set of CRDEs appears and has to be solved. To the best of our knowledge, solvability of the CRDEs appearing in the LQ differential game approach to the formation control problem has not been investigated in previous studies. In order to solve these equations, the formation control problem as the differential game is replaced by a discrete-time dynamic game. By using the LevyDesplanques theorem, the existence of a solution to the game associated set of CRDEs is derived. Furthermore, a backward iteration approximate solution to the CRDEs is presented by using the Gerschgorin's disc theorem and the matrix convergence criteria based on the spectral radius. An illustrative example is given to justify the models and solution. To demonstrate the accuracy of the presented approximate solution, a comparison is made with the results obtained by the ordinary differential equation (ODE) solver in the Matlab platform. Consequently, the contributions of this paper are twofold. First, solvability of the CRDEs of the formation control problem is proved. Second, a backward iteration approximate solution to the CRDEs is presented.

The remainder of this paper is organized as follows. The formation control problem formulation as the differential game is given in Section 2. The problem is discretized in Section 3. The main results of this paper are given in Section 4. An illustrative example is presented in Section 5. Section 6 is the conclusions.

\section{Problem definition}

Consider a networked system of $m$ mobile robots, each of which is described by a double integrator dynamics. The system dynamics can be expressed by

$$
\dot{\boldsymbol{z}}=\boldsymbol{A} \boldsymbol{z}+\sum_{i=1}^{m} \boldsymbol{B}_{i} \boldsymbol{u}_{i}, \quad \boldsymbol{z}(0)=\boldsymbol{z}_{0}
$$

with $\boldsymbol{A}=\left[\begin{array}{cc}\mathbf{0} & \boldsymbol{I}_{n m} \\ \mathbf{0} & \mathbf{0}\end{array}\right] \in \mathbf{R}^{2 n m+1}, \boldsymbol{B}_{i}=\left[\mathbf{0}_{n \times n m}, \mathbf{0}_{n \times 1}\right.$, $\left.\boldsymbol{b}_{i}^{\mathrm{T}}\right]^{\mathrm{T}} \in \mathbf{R}^{(2 n m+1) \times n}, \quad \boldsymbol{b}_{i}=\left[0 \cdots \boldsymbol{I}_{n} \cdots 0\right]^{\mathrm{T}} \in$ $\mathbf{R}^{n m \times n}$ where $\boldsymbol{I}_{n} \in \mathbf{R}^{n}$ is the identity matrix, $\boldsymbol{z}=$ $\left[\boldsymbol{q}_{1}^{\mathrm{T}}, \ldots, \boldsymbol{q}_{m}^{\mathrm{T}}, 1, \boldsymbol{v}_{1}^{\mathrm{T}}, \ldots, \boldsymbol{v}_{m}^{\mathrm{T}}\right]^{\mathrm{T}} \in \mathbf{R}^{2 n m+1}$ is a system state vector, $\boldsymbol{z}_{0}$ is a given initial state vector and $\boldsymbol{q}_{i}, \boldsymbol{v}_{i}, \boldsymbol{u}_{i} \in \mathbf{R}^{n}$ $(i=1, \ldots, m)$ are $n$-dimensional coordinates, velocity and control vectors, respectively. Superscript $\mathrm{T}$ denotes vector/matrix transpose.

A directed graph $\mathcal{G}=(\mathcal{V}, \mathcal{E})$ consists a set of vertices $\mathcal{V}$ and a set of edges $\mathcal{E}$ containing ordered pairs of distinct vertices. For the formation control problem, the set of vertices $\mathcal{V}=\{1,2, \ldots, m\}$ corresponds to the robots in the formation and then the set of edges $\mathcal{E} \subseteq\{(i, j): i, j \in \mathcal{V}\}$ represents the interconnections. Each edge $(i, j) \in \mathcal{E}$ is assigned with a weight $\mu_{i j}>0$. The set of neighbors of the robot $i$ in the graph topology is defined by $N_{i}=\{j \in \mathcal{V}:(i, j) \in \mathcal{E}\}$.

Assumption 1 Formation graph $\mathcal{G}$ is connected, i.e., for every pair of vertices $i, j \in \mathcal{V}$, from $i$ to $j$ for all $j=1, \ldots, m, j \neq i$, and there exists a path of (undirected) edges from $\mathcal{E}$.

For a robot $i$, the graph Laplacian $\boldsymbol{L}_{i}$ is defined as $\boldsymbol{L}_{i}=\boldsymbol{D} \boldsymbol{W}_{i} \boldsymbol{D}^{\mathrm{T}} \in \mathbf{R}^{m}$ where $\boldsymbol{D} \in \mathbf{R}^{m \times|\mathcal{E}|}$ is the incidence matrix and $\boldsymbol{W}_{i}=\operatorname{diag}\left(0, \ldots, \mu_{i j}, \ldots, 0\right) \in \mathbf{R}^{|\mathcal{E}|}$, $j \in N_{i}$ is a diagonal weight matrix. $\boldsymbol{D}$ 's $u v$ th element is 1 if the node $u$ is the head of the edge $v,-1$ if the node $u$ is the tail, and 0 , otherwise. The $n$-dimensional graph Laplacian $\mathcal{L}_{i}$ can be defined as $\mathcal{L}_{i}=\boldsymbol{L}_{i} \otimes \boldsymbol{I}_{n} \in \mathbf{R}^{n m}$ where $\otimes$ is the Kronecker product. Based on the properties of the Kronecker product [26], $\mathcal{L}_{i}$ can be rearranged as

$$
\begin{gathered}
\mathcal{L}_{i}=\boldsymbol{D} \boldsymbol{W}_{i} \boldsymbol{D}^{\mathrm{T}} \otimes \boldsymbol{I}_{n}= \\
\left(\boldsymbol{D} \otimes \boldsymbol{I}_{n}\right)\left(\boldsymbol{W}_{i} \otimes \boldsymbol{I}_{n}\right)\left(\boldsymbol{D} \otimes \boldsymbol{I}_{n}\right)^{\mathrm{T}}=\mathcal{D} \mathcal{W}_{i} \mathcal{D}^{\mathrm{T}}
\end{gathered}
$$

where $\mathcal{D}=\boldsymbol{D} \otimes \boldsymbol{I}_{n}$ and $\boldsymbol{W}_{i}=\boldsymbol{W}_{i} \otimes \boldsymbol{I}_{n}$. Let $\boldsymbol{q}=$ $\left[\boldsymbol{q}_{1}^{\mathrm{T}}, \ldots, \boldsymbol{q}_{m}^{\mathrm{T}}\right]^{\mathrm{T}}$ and $\boldsymbol{v}=\left[\boldsymbol{v}_{1}^{\mathrm{T}}, \ldots, \boldsymbol{v}_{m}^{\mathrm{T}}\right]^{\mathrm{T}}$. The graph Laplacian $\mathcal{L}_{i}$ is symmetric, positive semidefinite, and holds the property of sum-of-squares

$$
\begin{aligned}
\boldsymbol{v}^{\mathrm{T}} \mathcal{L}_{i} \boldsymbol{v} & =\sum_{(i, j) \in \mathcal{E}} \omega_{i j}\left\|\boldsymbol{q}_{i}-\boldsymbol{q}_{j}\right\|^{2} \\
\boldsymbol{q}^{\mathrm{T}} \mathcal{L}_{i} \boldsymbol{q} & =\sum_{(i, j) \in \mathcal{E}} \omega_{i j}\left\|\boldsymbol{v}_{i}-\boldsymbol{v}_{j}\right\|^{2}
\end{aligned}
$$

where $\|\cdot\|$ is the Euclidean norm in $\mathbf{R}^{n}$.

The formation requirement according to the formation graph can be expressed as

$$
\sum_{(i, j) \in \mathcal{E}} \mu_{i j}\left(\left\|\boldsymbol{q}_{i}-\boldsymbol{q}_{j}-\boldsymbol{d}_{i j}\right\|^{2}+\left\|\boldsymbol{v}_{i}-\boldsymbol{v}_{j}\right\|^{2}\right)
$$


where $\boldsymbol{d}_{i j} \in \mathbf{R}^{n}$ is the desired distance vector between two neighbor robots $i$ and $j$. Using the property of sum-ofsquares, (4) can be transformed into a matrix form:

$$
\begin{gathered}
\sum_{(i, j) \in \mathcal{E}}\left(\omega_{i j}\left\|\boldsymbol{q}_{i}-\boldsymbol{q}_{j}\right\|^{2}-2 \omega_{i j}\left(\boldsymbol{q}_{i}-\boldsymbol{q}_{j}\right)^{\mathrm{T}} \boldsymbol{d}_{i j}+\right. \\
\left.\omega_{i j}\left\|\boldsymbol{d}_{i j}\right\|^{2}+\omega_{i j}\left\|\boldsymbol{v}_{i}-\boldsymbol{v}_{j}\right\|^{2}\right)= \\
\boldsymbol{q}^{\mathrm{T}} \mathcal{L}_{i} \boldsymbol{q}-2 \boldsymbol{q}^{\mathrm{T}} \mathcal{D} \mathcal{W}_{i} \boldsymbol{d}+\boldsymbol{d}^{\mathrm{T}} \mathcal{W}_{i} \boldsymbol{d}+\boldsymbol{v}^{\mathrm{T}} \mathcal{L}_{i} \boldsymbol{v}=\boldsymbol{z}^{\mathrm{T}} \boldsymbol{Q}_{i} \boldsymbol{z}
\end{gathered}
$$

where

$$
\boldsymbol{Q}_{i}=\left[\begin{array}{ccc}
\mathcal{L}_{i} & -\mathcal{D} \mathcal{W}_{i} \boldsymbol{d} & \mathbf{0} \\
-\left(\mathcal{D} \mathcal{W}_{i} \boldsymbol{d}\right)^{\mathrm{T}} & \boldsymbol{d}^{\mathrm{T}} \mathcal{W}_{i} \boldsymbol{d} & \mathbf{0} \\
\mathbf{0} & \mathbf{0} & \mathcal{L}_{i}
\end{array}\right]
$$

$\boldsymbol{d}=\operatorname{col}\left(\boldsymbol{d}_{i j}\right)$ and $\operatorname{col}(\cdot)$ stands for "column vector". As $\boldsymbol{z}^{\mathrm{T}} \boldsymbol{Q}_{i} \boldsymbol{z} \geqslant 0$, the formation matrix $\boldsymbol{Q}_{i}$ is positive semidefinite.

The finite horizon quadratic cost function of the formation control for the robot $i$ is defined as

$$
J_{i}=\boldsymbol{z}\left(t_{f}\right)^{\mathrm{T}} \boldsymbol{Q}_{i f} \boldsymbol{z}\left(t_{f}\right)+\int_{0}^{t_{f}}\left(\boldsymbol{z}^{\mathrm{T}} \boldsymbol{Q}_{i} \boldsymbol{z}+\sum_{j=1}^{m} \boldsymbol{u}_{j}^{\mathrm{T}} \boldsymbol{R}_{i j} \boldsymbol{u}_{j}\right) \mathrm{d} t
$$

where

$$
\boldsymbol{Q}_{i f}=\left[\begin{array}{ccc}
\mathcal{L}_{i f} & -\mathcal{D} \mathcal{W}_{i f} \boldsymbol{d} & \mathbf{0} \\
-\left(\mathcal{D} \mathcal{W}_{i f} \boldsymbol{d}\right)^{\mathrm{T}} & \boldsymbol{d}^{\mathrm{T}} \mathcal{W}_{i f} \boldsymbol{d} & \mathbf{0} \\
\mathbf{0} & \mathbf{0} & \mathcal{L}_{i f}
\end{array}\right]
$$

$\mathcal{L}_{i f}=\mathcal{D} \mathcal{W}_{i f} \mathcal{D}^{\mathrm{T}}, \mathcal{L}_{i f}=\boldsymbol{W}_{i f} \otimes \boldsymbol{I}_{n}, \boldsymbol{W}_{i f}=$ $\operatorname{diag}\left(0, \ldots, \omega_{i j}, \ldots, 0\right) \in N_{i}, \omega_{i j}>0 . t_{f}$ is the finite time horizon and $\boldsymbol{R}_{i i}$ is a symmetric positive definite weight matrix. The formation control objective for the robot $i$ is to design $\boldsymbol{u}_{i}$ to minimize its cost function $J_{i}$ for the underlying system dynamics (1).

As there are $m$ different cost functions, the classical optimal control theory cannot be used to find the optimal control. Instead, the derivation of control strategies can be approached using the differential game theory. Under the open-loop control structure, the open-loop Nash equilibrium solution can be used as the formation control strategies. In the open-loop information structure, all players make their decisions based on the initial state. A Nash equilibrium is a strategy combination of all players (in the game) with the property that no one can gain lower cost by unilaterally deviating from it. The open-loop Nash equilibrium is defined as a set of admissible actions $\left(\boldsymbol{u}_{1}^{*}, \ldots, \boldsymbol{u}_{m}^{*}\right)$ if for all admissible $\left(\boldsymbol{u}_{1}, \ldots, \boldsymbol{u}_{m}\right)$ the following inequality

$$
\begin{gathered}
J^{i}\left(\boldsymbol{u}_{1}^{*}, \ldots, \boldsymbol{u}_{i-1}^{*}, \boldsymbol{u}_{i}^{*}, \boldsymbol{u}_{i+1}^{*}, \ldots, \boldsymbol{u}_{m}^{*}\right) \leqslant \\
J^{i}\left(\boldsymbol{u}_{1}^{*}, \ldots, \boldsymbol{u}_{i-1}^{*}, \boldsymbol{u}_{i}, \boldsymbol{u}_{i+1}^{*}, \ldots, \boldsymbol{u}_{m}^{*}\right)
\end{gathered}
$$

holds for $i=1, \ldots, m$ where $\boldsymbol{u}_{i} \in \Gamma_{i}$ and $\Gamma_{i}$ is the admissible strategy set for the player $i$. We assume that $\boldsymbol{u}_{i}$ consists of the set of measurable functions from $\left[0, t_{f}\right]$ into $\Gamma_{i}$ for which the differential equation (1) has a unique solution and the cost function (6) exists.

The standard solution for the above LQ differential game, or in other words, the formation control problem, is given in the following theorem (see [12] and [19]).

Theorem 1 For the formation control defined as a noncooperative LQ differential game by (1) and (6), let there exist a solution set $\boldsymbol{P}_{i}(i=1, \ldots, m)$ to the CRDE

$$
\begin{gathered}
\dot{\boldsymbol{P}}_{i}+\boldsymbol{P}_{i} \boldsymbol{A}-\boldsymbol{P}_{i} \sum_{j=1}^{m} \boldsymbol{S}_{j} \boldsymbol{P}_{j}+\boldsymbol{Q}_{i}+\boldsymbol{A}^{\mathrm{T}} \boldsymbol{P}_{i}=0, \\
\boldsymbol{P}_{i}\left(t_{f}\right)=\boldsymbol{Q}_{i f}
\end{gathered}
$$

where $\boldsymbol{S}_{i}=\boldsymbol{B}_{i} \boldsymbol{R}_{i i}^{-1} \boldsymbol{E}_{i}^{m}$. Then, there exists a unique openloop Nash equilibrium solution for the formation control given by

$$
\boldsymbol{u}_{i}=-\boldsymbol{R}_{i i}^{-1} \boldsymbol{B}_{i}^{\mathrm{T}} \boldsymbol{P}_{i} \boldsymbol{z} .
$$

To the best of our knowledge, there is no known set of conditions (on the parameters of the non-cooperative LQ game) that would guarantee the existence of a solution to CRDEs. Furthermore, there is no general analytic method for finding solutions to CRDEs; they have to be solved numerically by using terminal values and the backward iteration.

\section{Discretization of the problem}

In order to solve the CRDEs (8), the continuous time should be discretized into a series of time intervals. We divide the interval $\left[0, t_{f}\right]$ into $K$ equal intervals $\left[0, t_{1}\right],\left[t_{1}, t_{2}\right], \ldots,\left[t_{K-1}, t_{K}=t_{f}\right]$. The interval length (or sample time) is $t_{k}-t_{k-1}=\delta$ and $K$ is an even number. The control input variables are piecewise continuous. The system can thus be converted into the following discretetime model [27]:

$$
\boldsymbol{z}_{k+1}=\widetilde{\boldsymbol{A}} z_{k}+\sum_{i=1}^{m} \widetilde{\boldsymbol{B}}_{i} \boldsymbol{u}_{k}^{i}, \quad k=0, \ldots, K-1
$$

where $\widetilde{\boldsymbol{A}}=\mathrm{e}^{\delta \boldsymbol{A}}, \widetilde{\boldsymbol{B}}_{i}=\int_{0}^{\delta} \mathrm{e}^{t \boldsymbol{A}} \mathrm{d} t \boldsymbol{B}_{i}$.

The control effort part of the cost function (6) can be rewritten as

$$
\begin{gathered}
\sum_{j=1}^{m} \int_{0}^{t_{f}} \boldsymbol{u}_{j}^{\mathrm{T}} \boldsymbol{R}_{i j} \boldsymbol{u}_{j} \mathrm{~d} t=\delta \sum_{j=1}^{m} \sum_{k=0}^{K-1} \boldsymbol{u}_{k}^{j^{\mathrm{T}}} \boldsymbol{R}_{i j} \boldsymbol{u}_{k}^{j}= \\
\sum_{j=1}^{m} \sum_{k=0}^{K-1} \boldsymbol{u}_{k}^{j^{\mathrm{T}}} \widetilde{\boldsymbol{R}}_{i j} \boldsymbol{u}_{k}^{j}
\end{gathered}
$$

where $\widetilde{\boldsymbol{R}}_{i j}=\delta \boldsymbol{R}_{i j}$. Since the control signals are unit-step, (11) is not an approximation. The rest of the cost function 
(6) can be approximated using Simpson's rule for the numerical approximation of definite integrals as follows:

$$
\begin{gathered}
\boldsymbol{z}\left(t_{f}\right)^{\mathrm{T}} \boldsymbol{Q}_{i f} \boldsymbol{z}\left(t_{f}\right)+\int_{0}^{t_{f}} \boldsymbol{z}^{z} T \boldsymbol{Q}_{i} \boldsymbol{z} \mathrm{d} t \approx \\
\delta \sum_{k=0}^{K} \boldsymbol{z}_{k}^{\mathrm{T}} \boldsymbol{Q}_{i}^{k} \boldsymbol{z}_{k}=\sum_{k=0}^{K} \boldsymbol{z}_{k}^{\mathrm{T}} \widetilde{\boldsymbol{Q}}_{i}^{k} \boldsymbol{z}_{k}
\end{gathered}
$$

where $\boldsymbol{Q}_{i}^{0}=\frac{1}{3} \boldsymbol{Q}_{i}, \boldsymbol{Q}_{i}^{1,3, \ldots, K-1}=\frac{4}{3} \boldsymbol{Q}_{i}, \boldsymbol{Q}_{i}^{2,4, \ldots, K-2}=$ $\frac{2}{3} \boldsymbol{Q}_{i}, \boldsymbol{Q}_{i}^{K}=\frac{1}{3} \boldsymbol{Q}_{i}+\boldsymbol{Q}_{i f}, \widetilde{\boldsymbol{Q}}_{i}^{k}=\delta \boldsymbol{Q}_{i}^{k}$. By combining (11) and (12), the cost function (6) can be discretized (and approximated) by

$$
\begin{array}{r}
\mathcal{J}^{i}=\sum_{k=0}^{K} \boldsymbol{z}_{k}^{\mathrm{T}} \widetilde{\boldsymbol{Q}}_{i}^{k} \boldsymbol{z}_{k}+\sum_{j=1}^{m} \sum_{k=0}^{K-1} \boldsymbol{u}_{k}^{j^{\mathrm{T}}} \widetilde{\boldsymbol{R}}_{i j} \boldsymbol{u}_{k}^{j}= \\
\boldsymbol{z}_{K}^{\mathrm{T}} \widetilde{\boldsymbol{Q}}_{i}^{K} \boldsymbol{z}_{K}+\sum_{k=0}^{K-1}\left(\boldsymbol{z}_{k}^{\mathrm{T}} \widetilde{\boldsymbol{Q}}_{i}^{k} \boldsymbol{z}_{k}+\sum_{j=1}^{m} \boldsymbol{u}_{k}^{j^{\mathrm{T}}} \widetilde{\boldsymbol{R}}_{i j} \boldsymbol{u}_{k}^{j}\right) .
\end{array}
$$

Remark 1 We observe that $\boldsymbol{A}^{2}=\mathbf{0}$. Then the matrix exponential $\mathrm{e}^{t \boldsymbol{A}}$ and $\mathrm{e}^{t \boldsymbol{A}^{\mathrm{T}}}$ are $\mathrm{e}^{t \boldsymbol{A}}=\boldsymbol{I}+t \boldsymbol{A}, \mathrm{e}^{t \boldsymbol{A}^{\mathrm{T}}}=$ $\boldsymbol{I}+t \boldsymbol{A}^{\mathrm{T}}$. Therefore, $\widetilde{\boldsymbol{A}}=\boldsymbol{I}+\delta \boldsymbol{A}, \widetilde{\boldsymbol{B}}_{i}=\delta \boldsymbol{B}_{i}+\frac{\delta^{2}}{2} \boldsymbol{A} \boldsymbol{B}_{i}$.

For the discretized formation control problem given as a discrete-time LQ dynamic game by (10) and (13), we can characterize the open-loop Nash equilibrium solution by the following theorem [28]. by

Theorem 2 Let $\boldsymbol{\Lambda}^{k}$ and $\boldsymbol{M}_{i}^{k+1}$ be matrices generated

$$
\begin{gathered}
\boldsymbol{M}_{i}^{k}=\widetilde{\boldsymbol{Q}}_{i}^{k}+\widetilde{\boldsymbol{A}}^{\mathrm{T}} \boldsymbol{M}_{i}^{k+1} \boldsymbol{\Lambda}_{k}^{-1} \widetilde{\boldsymbol{A}}, \quad \boldsymbol{M}_{i}^{K}=\widetilde{\boldsymbol{Q}}_{i}^{K} \\
\boldsymbol{\Lambda}_{k}=\boldsymbol{I}+\sum_{j=1}^{m} \widetilde{\boldsymbol{S}}_{j} \boldsymbol{M}_{j}^{k+1}
\end{gathered}
$$

where $\widetilde{\boldsymbol{S}}_{i}=\widetilde{\boldsymbol{B}}_{i} \widetilde{\boldsymbol{R}}_{i i}^{-1} \widetilde{\boldsymbol{B}}_{i}^{\mathrm{T}}$. If $\boldsymbol{\Lambda}_{k}$ is invertible, the game admits a unique open-loop Nash equilibrium solution given by

$$
\boldsymbol{u}_{k}^{i}=-\widetilde{\boldsymbol{R}}_{i i}^{-1} \widetilde{\boldsymbol{B}}_{i}^{\mathrm{T}} \boldsymbol{M}_{i}^{k+1} \boldsymbol{\Lambda}_{k}^{-1} \widetilde{\boldsymbol{A}} \boldsymbol{z}_{k}
$$

where the corresponding state trajectory is generated by $\boldsymbol{z}_{k+1}=\boldsymbol{\Lambda}_{k}^{-1} \widetilde{\boldsymbol{A}} \boldsymbol{z}_{k}$.

As it can be seen the necessary and sufficient condition for the existence of the Riccati difference equations (coupled through $\boldsymbol{\Lambda}_{k}$ ) is invertibility of $\boldsymbol{\Lambda}_{k}$. In this case, the Nash equilibrium controls of discrete-time formation control exist.

\section{Main results}

In this section, the main results of this paper are presented, but the following definition is introduced first.
Definition 1 Matrix $\boldsymbol{X} \in \mathbf{R}^{n}$ is strictly diagonally dominant if for all $i=1, \ldots, n,\left|X_{i i}\right|>\sum_{j \neq i}\left|X_{i j}\right|$.

The well-known Levy-Desplanques theorem states that strictly diagonally dominant matrices are nonsingular.

Theorem 3 For a non-large $t_{f}$, the matrix $\boldsymbol{\Lambda}_{k}(k=$ $0, \ldots, K-1)$ is non-singular.

Proof By using terminal values and the backward iteration,

$$
\boldsymbol{\Lambda}_{K-1}=\boldsymbol{I}+\sum_{j=1}^{m} \widetilde{\boldsymbol{S}}_{j} \boldsymbol{M}_{j}^{K}=\boldsymbol{I}+\sum_{j=1}^{m} \widetilde{\boldsymbol{S}}_{j} \widetilde{\boldsymbol{Q}}_{j}^{K}
$$

where also,

$$
\sum_{j=1}^{m} \widetilde{\boldsymbol{S}}_{j} \widetilde{\boldsymbol{Q}}_{j}^{K}=\left[\begin{array}{ccc}
\frac{\delta^{4}}{4} \boldsymbol{H} & \frac{\delta^{4}}{4} \boldsymbol{V} & \frac{\delta^{3}}{2} \boldsymbol{H} \\
0 & 0 & 0 \\
\frac{\delta^{3}}{2} \boldsymbol{H} & \frac{\delta^{3}}{2} \boldsymbol{V} & \delta^{2} \boldsymbol{H}
\end{array}\right]
$$

with

$$
\begin{gathered}
\boldsymbol{H}=\sum_{j=1}^{m} \boldsymbol{b}_{j} \boldsymbol{R}_{j j}^{-1} \boldsymbol{b}_{j}^{\mathrm{T}}\left(\frac{1}{3} \mathcal{L}_{j}+\mathcal{L}_{j f}\right) \\
\boldsymbol{V}=\sum_{j=1}^{m} \boldsymbol{b}_{j} \boldsymbol{R}_{j j}^{-1} \boldsymbol{b}_{j}^{\mathrm{T}} \mathcal{D}\left(-\frac{1}{3} \mathcal{W}_{j}-\mathcal{W}_{j f}\right) \boldsymbol{d} .
\end{gathered}
$$

From Remark 1, we have

$$
\begin{gathered}
\widetilde{\boldsymbol{A}}=\left[\begin{array}{ccc}
\boldsymbol{I}_{n m} & \mathbf{0} & \delta \boldsymbol{I}_{n m} \\
\mathbf{0} & 1 & \mathbf{0} \\
\mathbf{0}_{n m} & \mathbf{0} & \boldsymbol{I}_{n m}
\end{array}\right], \quad \widetilde{\boldsymbol{B}}_{i}=\left[\begin{array}{c}
\frac{\delta^{2}}{2} \boldsymbol{b}_{i} \\
0 \\
\delta \boldsymbol{b}_{i}
\end{array}\right], \\
\widetilde{\boldsymbol{S}}_{i}=\left[\begin{array}{cccc}
\frac{\delta^{3}}{4} \boldsymbol{b}_{i} \boldsymbol{R}_{i i}^{-1} \boldsymbol{b}_{i}^{\mathrm{T}} & \mathbf{0} & \frac{\delta^{2}}{2} \boldsymbol{b}_{i} \boldsymbol{R}_{i i}^{-1} \boldsymbol{b}_{i}^{\mathrm{T}} \\
\mathbf{0} & 0 & \mathbf{0} \\
\frac{\delta^{2}}{2} \boldsymbol{b}_{i} \boldsymbol{R}_{i i}^{-1} \boldsymbol{b}_{i}^{\mathrm{T}} & \mathbf{0} & \delta \boldsymbol{b}_{i} \boldsymbol{R}_{i i}^{-1} \boldsymbol{b}_{i}^{\mathrm{T}}
\end{array}\right] .
\end{gathered}
$$

Also,

$$
\begin{gathered}
\widetilde{\boldsymbol{Q}}_{j}^{K}=\delta \boldsymbol{Q}_{j}^{K}=\delta\left(\frac{1}{3} \boldsymbol{Q}_{j}+\boldsymbol{Q}_{j f}\right)= \\
{\left[\begin{array}{ccc}
\delta \mathcal{X}_{j} & \delta \mathcal{D}\left(-\mathcal{Y}_{j}\right) \boldsymbol{d} & \mathbf{0} \\
\delta\left(\mathcal{D}\left(-\mathcal{Y}_{j}\right) \boldsymbol{d}\right)^{\mathrm{T}} & \delta \boldsymbol{d}^{\mathrm{T}} \mathcal{Y}_{j} \boldsymbol{d} & \mathbf{0} \\
\mathbf{0} & \mathbf{0} & \delta \mathcal{X}_{j}
\end{array}\right]}
\end{gathered}
$$

where $\mathcal{X}_{j}=\frac{1}{3} \mathcal{L}_{j}+\mathcal{L}_{j f}, \mathcal{Y}_{j}=\frac{1}{3} \mathcal{W}_{j}+\mathcal{W}_{j f}$, and therefore, it is confirmed that $\sum_{j=1}^{m} \widetilde{\boldsymbol{S}}_{j} \widetilde{\boldsymbol{Q}}_{j}^{K}$ has the above form.

On the other hand, the definition of the Laplacian matrix will lead to the following matrix form of $\mathcal{L}_{i}$ and $\mathcal{L}_{i f}$ : 


$$
\mathcal{L}_{i}=\left[\begin{array}{ccccc}
\mu_{i, 1} & \cdots & -\mu_{i, 1} & \cdots & 0 \\
\vdots & \ddots & \vdots & \ddots & \vdots \\
-\mu_{i, 1} & \cdots & \sum_{(i, k) \in \mathcal{E}} \mu_{i, k} & \cdots & -\mu_{i, m} \\
\vdots & \ddots & \vdots & \ddots & \vdots \\
0 & \cdots & -\mu_{i, m} & \cdots & \mu_{i, m}
\end{array}\right] \otimes \boldsymbol{I}_{n}, \quad \mathcal{L}_{i f}=\left[\begin{array}{ccccc}
\omega_{i, 1} & \cdots & -\omega_{i, 1} & \cdots & 0 \\
\vdots & \ddots & \vdots & \ddots & \vdots \\
-\omega_{i, 1} & \cdots & \sum_{(i, k) \in \mathcal{E}} \omega_{i, k} & \cdots & -\omega_{i, m} \\
\vdots & \ddots & \vdots & \ddots & \vdots \\
0 & \cdots & -\omega_{i, m} & \cdots & \omega_{i, m}
\end{array}\right] \otimes \boldsymbol{I}_{n}
$$

Also, we notice that

$$
\mathcal{D} \mathcal{W}_{i} \boldsymbol{d}=\left[\begin{array}{c}
\left(\mu_{i, 1} \otimes \boldsymbol{I}_{n}\right) \boldsymbol{d}_{i, 1} \\
\vdots \\
-\sum_{(i, k) \in \mathcal{E}}\left(\mu_{i, k} \otimes \boldsymbol{I}_{n}\right) \boldsymbol{d}_{i, k} \\
\vdots \\
\left(\mu_{i, m} \otimes \boldsymbol{I}_{n}\right) \boldsymbol{d}_{i, m}
\end{array}\right] .
$$

Based on these matrix structures, we have

$$
\begin{aligned}
& \sum_{j=1}^{m} \boldsymbol{b}_{j} \boldsymbol{R}_{j j}^{-1} \boldsymbol{b}_{j}^{\mathrm{T}}\left(\frac{1}{3} \mathcal{L}_{j}+\mathcal{L}_{j f}\right)=\operatorname{diag}\left(\boldsymbol{R}_{11}^{-1}, \ldots, \boldsymbol{R}_{m m}^{-1}\right) \times \\
& {\left[\begin{array}{ccccc}
\sum_{(1, k) \in \mathcal{E}}\left(\frac{1}{3} \mu_{1, k}+\omega_{1, k}\right) & \cdots & \left(-\frac{1}{3} \mu_{1, k}-\omega_{1, k}\right) & \cdots & \left(-\frac{1}{3} \mu_{1, m}-\omega_{1, m}\right) \\
\vdots & \ddots & \vdots
\end{array}\right.} \\
& \left.\begin{array}{ccccc}
\left(-\frac{1}{3} \mu_{i, 1}-\omega_{i, 1}\right) & \ldots & \sum_{(i, k) \in \mathcal{E}}\left(\frac{1}{3} \mu_{i, k}+\omega_{i, k}\right) & \cdots & \left(-\frac{1}{3} \mu_{i, m}-\omega_{i, m}\right) \\
\vdots & \ddots & \vdots & \ddots & \vdots \\
\left(-\frac{1}{3} \mu_{m, 1}-\omega_{m, 1}\right) & \ldots & \left(-\frac{1}{3} \mu_{m, k}-\omega_{m, k}\right) & \cdots & \sum_{(1, k) \in \mathcal{E}}\left(\frac{1}{3} \mu_{m, k}+\omega_{m, k}\right)
\end{array}\right] \otimes \boldsymbol{I}_{n} \\
& \sum_{j=1}^{m} \boldsymbol{b}_{j} \boldsymbol{R}_{j j}^{-1} \boldsymbol{b}_{j}^{\mathrm{T}} \mathcal{D}\left(-\frac{1}{3} \mathcal{W}_{j}-\mathcal{W}_{j f}\right) \boldsymbol{d}= \\
& \delta^{3} \sum_{(i, k) \in \mathcal{E}}\left(\frac{1}{3} \mu_{i, k}+\omega_{i, k}\right)\left(1+\frac{\delta}{4}\left|\left[\boldsymbol{d}_{i, k}\right]_{j}\right|\right)<\left[\boldsymbol{R}_{i i}\right]_{j} \\
& \operatorname{diag}\left(\boldsymbol{R}_{11}^{-1}, \ldots, \boldsymbol{R}_{m m}^{-1}\right) \times \\
& {\left[\begin{array}{c}
\sum_{(1, k) \in \mathcal{E}}\left(\left(\frac{1}{3} \mu_{1, k}+\omega_{1, k}\right) \otimes \boldsymbol{I}_{n}\right) \boldsymbol{d}_{1, k} \\
\vdots \\
\sum_{(i, k) \in \mathcal{E}}\left(\left(\frac{1}{3} \mu_{i, k}+\omega_{i, k}\right) \otimes \boldsymbol{I}_{n}\right) \boldsymbol{d}_{i, k} \\
\vdots \\
\sum_{(m, k) \in \mathcal{E}}\left(\left(\frac{1}{3} \mu_{m, k}+\omega_{m, k}\right) \otimes \boldsymbol{I}_{n}\right) \boldsymbol{d}_{m, k}
\end{array}\right] .}
\end{aligned}
$$


As the result of Theorem 3, the existence of Nash equilibrium controls for the discrete-time formation control problem now is concluded. The next theorem demonstrates an approximate solution to the CRDEs (8).

Lemma 1 Let $G \in \mathbf{R}^{n}$ be a square matrix, and assume that $\operatorname{sprad}(\boldsymbol{G})<1$ that $\operatorname{sprad}(\boldsymbol{G})$ stands for "spectral radius of $G$ ". The series $\sum_{f=0}^{\infty} G^{f}$ converges absolutely, and $(I-G)^{-1}=\sum_{f=0}^{\infty} \boldsymbol{G}^{f}$.

We refer the reader to [29], Proposition 9.4.13 for this lemma.

Theorem 4 For the $m$-robot formation control defined as a finite horizon LQ differential game by (1) and (6), the approximate solution of the CRDEs (8) at the $k$ th sample time is given as follows:

$$
\boldsymbol{P}_{k}^{i} \approx\left(\widetilde{\boldsymbol{A}}^{\mathrm{T}}\right)^{\frac{1}{2}} \boldsymbol{M}_{i}^{k+1}\left(\boldsymbol{I}-\sum_{j=1}^{m} \widetilde{\boldsymbol{S}}_{j} \boldsymbol{M}_{j}^{k+1}\right) \widetilde{\boldsymbol{A}}
$$

where $\boldsymbol{M}_{i}^{k}$ by using terminal values and the backward iteration can be calculated by

$$
\begin{gathered}
\boldsymbol{M}_{i}^{k}=\widetilde{\boldsymbol{Q}}_{i}^{k}+\widetilde{\boldsymbol{A}}^{\mathrm{T}} \boldsymbol{M}_{i}^{k+1}\left(\boldsymbol{I}-\sum_{j=1}^{m} \widetilde{\boldsymbol{S}}_{j} \boldsymbol{M}_{j}^{k+1}\right) \widetilde{\boldsymbol{A}}, \\
\boldsymbol{M}_{i}^{K}=\widetilde{\boldsymbol{Q}}_{i}^{K} .
\end{gathered}
$$

Proof Consider the matrix $\sum_{j=1}^{m} \widetilde{\boldsymbol{S}}_{j} \widetilde{\boldsymbol{Q}}_{j}^{K}$. We suppose that for $h=1, \ldots, n$,

$$
\begin{gathered}
\max \left[\left[\boldsymbol{R}^{-1} 11\right]_{h} \sum_{(1, k) \in \mathcal{E}}\left(\frac{1}{3} \mu_{1, k}+\omega_{1, k}\right), \ldots,\right. \\
{\left[\boldsymbol{R}^{-1} i i\right]_{h} \sum_{(i, k) \in \mathcal{E}}\left(\frac{1}{3} \mu_{i, k}+\omega_{i, k}\right), \ldots,} \\
\left.\left[\boldsymbol{R}^{-1} m m\right]_{h} \sum_{(m, k) \in \mathcal{E}}\left(\frac{1}{3} \mu_{m, k}+\omega_{m, k}\right)\right]= \\
{\left[\boldsymbol{R}^{-1} j j\right]_{h} \sum_{(j, k) \in \mathcal{E}}\left(\frac{1}{3} \mu_{j, k}+\omega_{j, k}\right) .}
\end{gathered}
$$

The location of the eigenvalues of a matrix is bounded by the Gerschgorin's disc theorem [30]. Applying the theorem to the matrix $\sum_{j=1}^{m} \widetilde{\boldsymbol{S}}_{j} \widetilde{\boldsymbol{Q}}_{j}^{K}$ gives that

$$
\operatorname{sprad}\left(\sum_{j=1}^{m} \widetilde{\boldsymbol{S}}_{j} \widetilde{\boldsymbol{Q}}_{j}^{K}\right)<\left[\boldsymbol{R}^{-1} j j\right]_{h}\left\{\left(\delta^{3}+\right.\right.
$$

$$
\begin{array}{r}
\left.2 \delta^{2}\right) \sum_{(j, k) \in \mathcal{E}}\left(\frac{1}{3} \mu_{j, k}+\omega_{j, k}\right)+\frac{\delta^{3}}{2} \mid\left(\sum _ { ( i , k ) \in \mathcal { E } } \left(\frac{1}{3} \mu_{j, k}+\right.\right. \\
\left.\left.\left.\omega_{j, k}\right) \otimes \boldsymbol{I}_{n}\right)\left[d_{j, k}\right]_{h} \mid\right\}
\end{array}
$$

This inequality is almost equivalent to the inequality (26), and therefore, here it can be concluded that $\operatorname{sprad}\left(\sum_{j=1}^{m} \widetilde{\boldsymbol{S}}_{j} \widetilde{\boldsymbol{Q}}_{j}^{K}\right)<1$. The same conclusion can be made for matrices $\sum_{j=1}^{m} \widetilde{\boldsymbol{S}}_{j} \widetilde{\boldsymbol{M}}_{j}^{K-1}, \ldots, \sum_{j=1}^{m} \widetilde{\boldsymbol{S}}_{j} \widetilde{\boldsymbol{M}}_{j}^{0}$. Based on this conclusion and according to Lemma 1, we have $\boldsymbol{\Lambda}_{k}^{-1}=\sum_{f=0}^{\infty}\left(-\sum_{j=1}^{m} \widetilde{\boldsymbol{S}}_{j} \boldsymbol{M}_{j}^{k+1}\right)^{f}$, where the series can be cut at $f=1 ; \boldsymbol{\Lambda}_{k}^{-1} \approx \boldsymbol{I}-\sum_{j=1}^{m} \widetilde{\boldsymbol{S}}_{j} \boldsymbol{M}_{j}^{k+1}$.

By substituting $\boldsymbol{\Lambda}_{k}^{-1}$ from above into (14), we can obtain (28). Also, by substituting $\widetilde{\boldsymbol{A}}$ and $\widetilde{\boldsymbol{B}}_{i}$ from Remark 1 into (16), we have

$$
\begin{gathered}
\boldsymbol{u}_{k}^{i} \approx-\frac{1}{\delta} \boldsymbol{R}_{i i}^{-1}\left(\delta \boldsymbol{B}_{i}^{\mathrm{T}}+\frac{\delta^{2}}{2} \boldsymbol{B}_{i}^{\mathrm{T}} \boldsymbol{A}^{\mathrm{T}}\right) \boldsymbol{M}_{i}^{k+1} \times \\
\left(\boldsymbol{I}-\sum_{j=1}^{m} \widetilde{\boldsymbol{S}}_{j} \boldsymbol{M}_{j}^{k+1}\right) \widetilde{\boldsymbol{A}} \boldsymbol{z}_{k}=-\boldsymbol{R}^{-1} i i \boldsymbol{B}_{i}^{\mathrm{T}}\left[\left(\boldsymbol{I}+\frac{\delta}{2} \boldsymbol{A}^{\mathrm{T}}\right) \times\right. \\
\left.\boldsymbol{M}_{i}^{k+1}\left(\boldsymbol{I}-\sum_{j=1}^{m} \widetilde{\boldsymbol{S}}_{j} \boldsymbol{M}_{j}^{k+1}\right) \widetilde{\boldsymbol{A}}\right] \boldsymbol{z}_{k}
\end{gathered}
$$

where (27) appears here by having regard to (9).

\section{Simulations}

Simulations have been performed to verify the models and the results derived in Theorem 3 and Theorem 4. The communication topology of the five robots in a desired triangle formation is defined by the incidence matrix

$$
\boldsymbol{D}=\left[\begin{array}{cccc}
-1 & -1 & 0 & 0 \\
1 & 0 & -1 & 0 \\
0 & 0 & 1 & 0 \\
0 & 1 & 0 & -1 \\
0 & 0 & 0 & 1
\end{array}\right]
$$

The weighting parameters of this formation are set according to Theorem 3 and shown in the form of the following matrices: $\boldsymbol{W}_{1}=\operatorname{diag}(1,1,0,0)$, $\boldsymbol{W}_{2}=\operatorname{diag}(1,0,1,0), \boldsymbol{W}_{3}=\operatorname{diag}(0,0,1,0), \boldsymbol{W}_{4}=$ $\operatorname{diag}(0,1,0,1), \boldsymbol{W}_{5}=\operatorname{diag}(0,0,0,1), \boldsymbol{W}_{i f}=5 \boldsymbol{W}_{i}(i=$ $1, \ldots, 5)$. 
The weight matrices for control effort in the cost functions are selected to be $\boldsymbol{R}_{i i}=10 \boldsymbol{I}_{2}(i=1, \ldots, 5)$. The finite horizon time and sampling time for discretization are selected to be $t_{f}=10 \mathrm{~s}$ and $\delta=0.1 \mathrm{~s}$. The desired offset vectors of the formation shape among the robots are set to be: $\boldsymbol{d}_{12}=\boldsymbol{d}_{23}=[-2,-4]^{\mathrm{T}}, \boldsymbol{d}_{14}=\boldsymbol{d}_{45}=[2,-4]^{\mathrm{T}}$. The initial positions of the five robots are assumed to be at $\boldsymbol{q}_{1}=[1,0]^{\mathrm{T}}, \boldsymbol{q}_{2}=[4,0]^{\mathrm{T}}, \boldsymbol{q}_{3}=[7,0]^{\mathrm{T}}, \boldsymbol{q}_{4}=[-1,0]^{\mathrm{T}}$, $\boldsymbol{q}_{5}=[-4,0]^{\mathrm{T}}$, and the initial velocities of the five robots are assumed to be $\boldsymbol{v}_{1}=[0,2]^{\mathrm{T}}, \boldsymbol{v}_{2}=[0,3]^{\mathrm{T}}, \boldsymbol{v}_{3}=$ $[0,1.5]^{\mathrm{T}}, \boldsymbol{v}_{4}=[0,1]^{\mathrm{T}}, \boldsymbol{v}_{5}=[0,2.5]^{\mathrm{T}}$.

The approximate solution of the CRDEs (8) for this triangle formation can be calculated through (27) and (28), and hence the approximate solution of the open-loop Nash equilibrium and the state trajectory for the five robots are determined according to (16).

The progression trajectories of the five robots in the triangle formation in the $x-y$ plane are demonstrated in Fig. 1. In the figure, the robots are shown in their initial positions at $t=0 \mathrm{~s}$, at time $t=3 \mathrm{~s}$, and at time $t=9.8 \mathrm{~s}$ right before the terminal time. Clearly, the robot's positions form the desired triangle formation shape at the specified terminal time $t_{f}$. The evolution of trajectories in this figure shows the robots acquire the desired relative positions with respect to each other given by the desired offset vectors of the formation shape, i.e., $\boldsymbol{d}_{12}, \boldsymbol{d}_{23}, \boldsymbol{d}_{14}, \boldsymbol{d}_{45}$. Fig. 2 shows the time histories of the robots' relative displacements according to $\boldsymbol{q}_{i}-\boldsymbol{q}_{j}-\boldsymbol{d}_{i j}$, relative velocities according to $\boldsymbol{v}_{i}-\boldsymbol{v}_{j}$ and control inputs $\boldsymbol{u}_{i}$. As can be seen in this figure the control objectives defined by (4) are achieved. Consequently, the validity of the models and the solution is obvious.

In order to measure the accuracy of the approximate solution to the CEDEs (8), we resolve these equations by the ODE45 solver in Matlab software. The approximate solution matrix for the robot $i=4$ at $t=0$ is denoted as $\boldsymbol{P}_{0}^{4}$ and the result of the ODE solver in the Matlab platform is denoted as $\boldsymbol{P}_{4}(0)$ and both are shown at the bottom of the page.

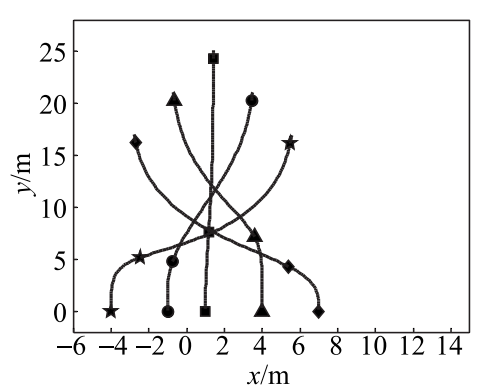

- : Robot $1 ; \quad \boldsymbol{\Delta}$ : Robot $2 ; \quad \bullet:$ Robot $3 ; \quad \bullet:$ Robot $4 ; \quad \star$ : Robot 5. Fig. 1 Motion trajectories of five robots in desired triangle formation acquisition

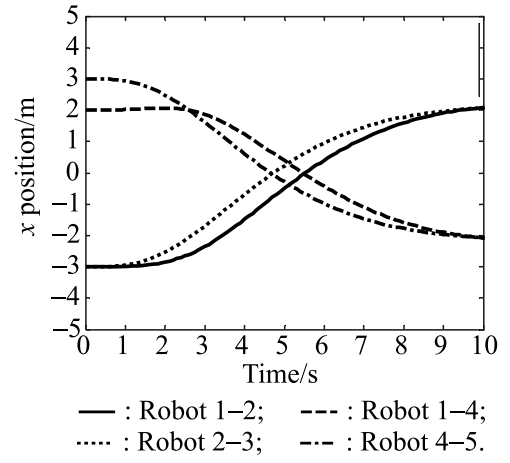

(a) Time histories of the relative displacements on the $x$ axis

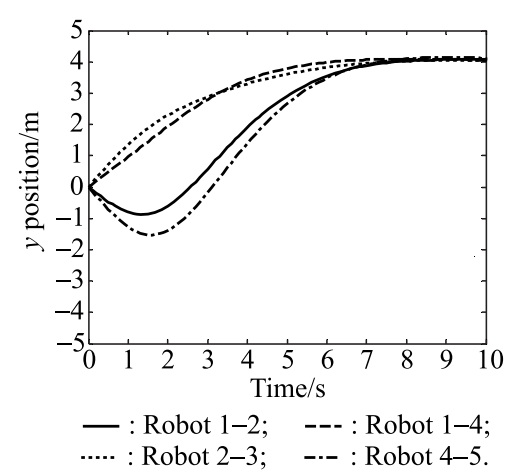

(b) Time histories of the relative displacements on the $y$ axis

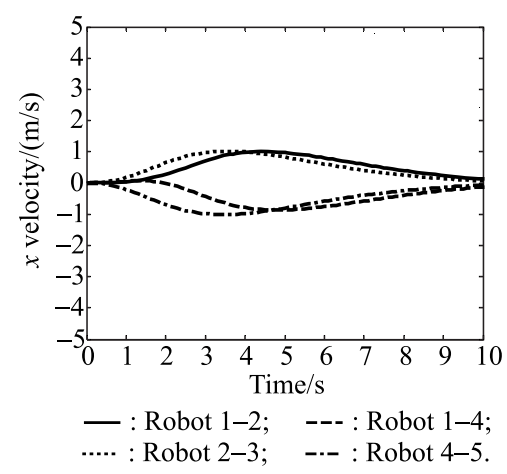

(c) Time histories of the relative velocities on the $x$ axis

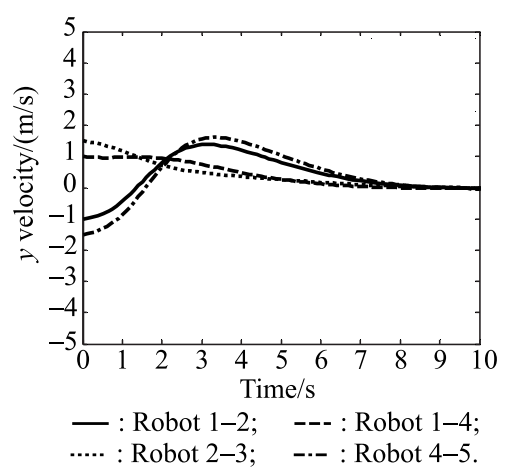

(d) Time histories of the relative velocities on the $y$ axis 


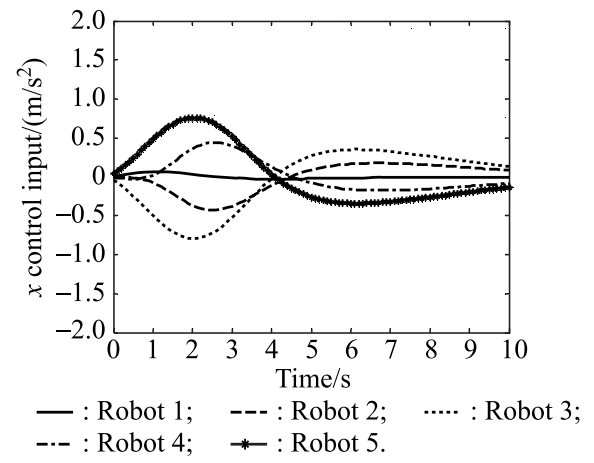

(e) Time histories of the control inputs on the $x$ axis

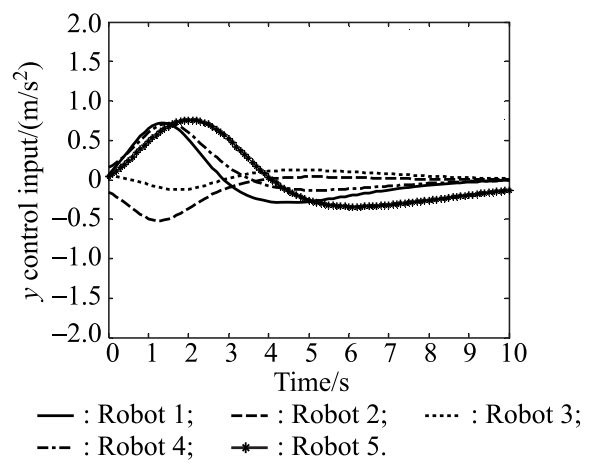

(f) Time histories of the control inputs on the $y$ axis

Fig. 2 Time histories of the relative displacements, relative velocities and control inputs on the $x$ and $y$ axis
The comparison with the results of the ODE solver in Matlab platform, it is seen that the approximate solution is fairly accurate and satisfactory. Moreover, the zero rows in the solution matrices indicate that the CRDEs (8) do not involve the information from non-neighbor robots. In other words, the Nash equilibrium controls (9) of the formation control problem only require local information from the neighbors on the communication topology.

\section{Conclusions}

In this paper, the formation control of multi-mobile robots is modeled as a non-cooperative LQ differential game. The continuous time is discretized into a series of time intervals, and thus the formation control problem is converted to a discrete-time problem. Sufficient conditions for the existence of Nash equilibrium controls of the discrete-time formation control problem are derived, and under these acquired conditions, an approximate solution for the CRDEs is presented. The simulation results demonstrate that the models are valid, and the solutions are fairly accurate and satisfactory. Future work includes considering the collision avoidance, in which formation cost functions are reconstructed to avoid collisions with obstacles and other robots. For this problem as a differential game, the feedback Nash equilibrium solution can be investigated.

$$
\begin{aligned}
& P_{0}^{4}=
\end{aligned}
$$

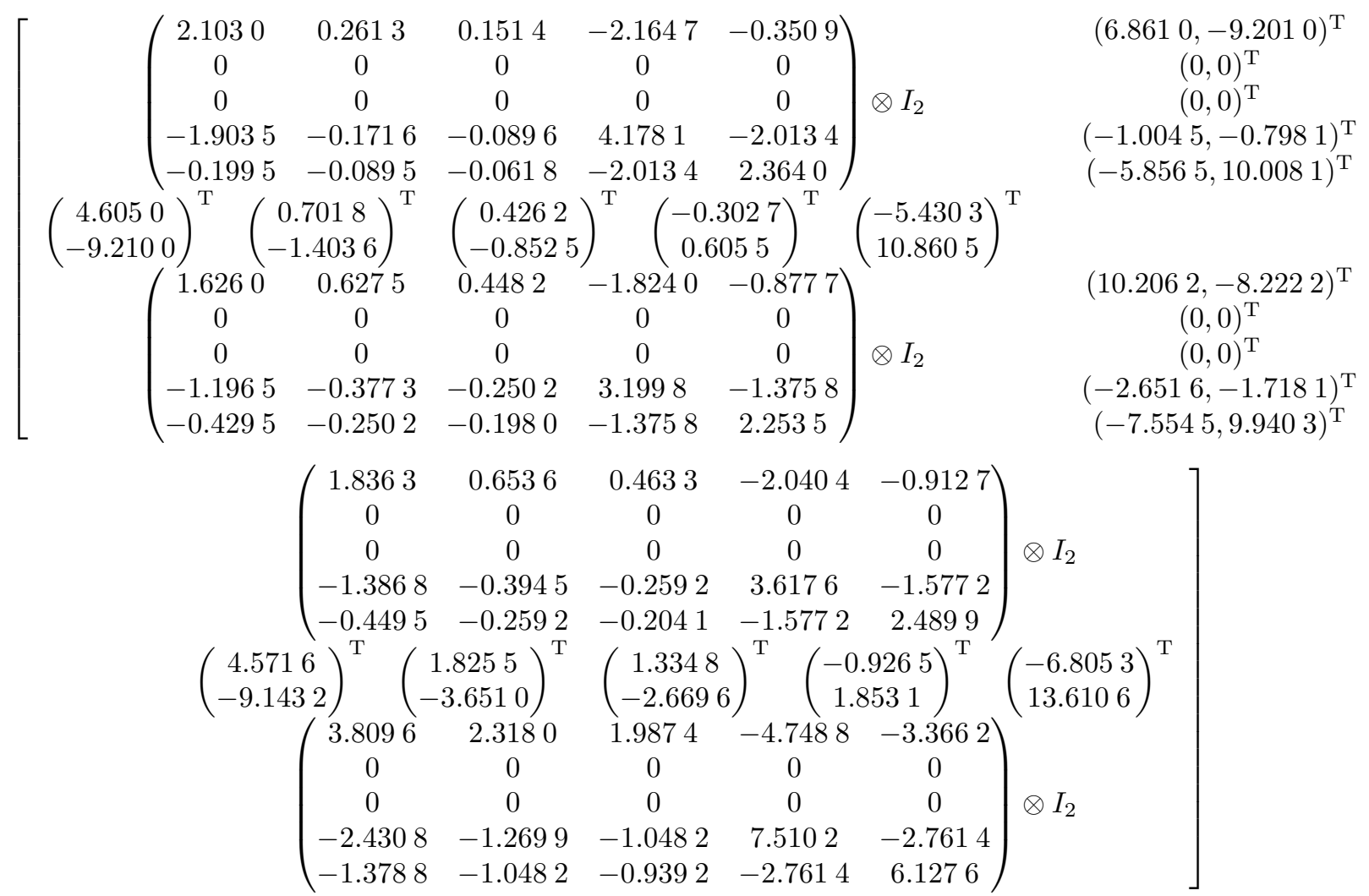




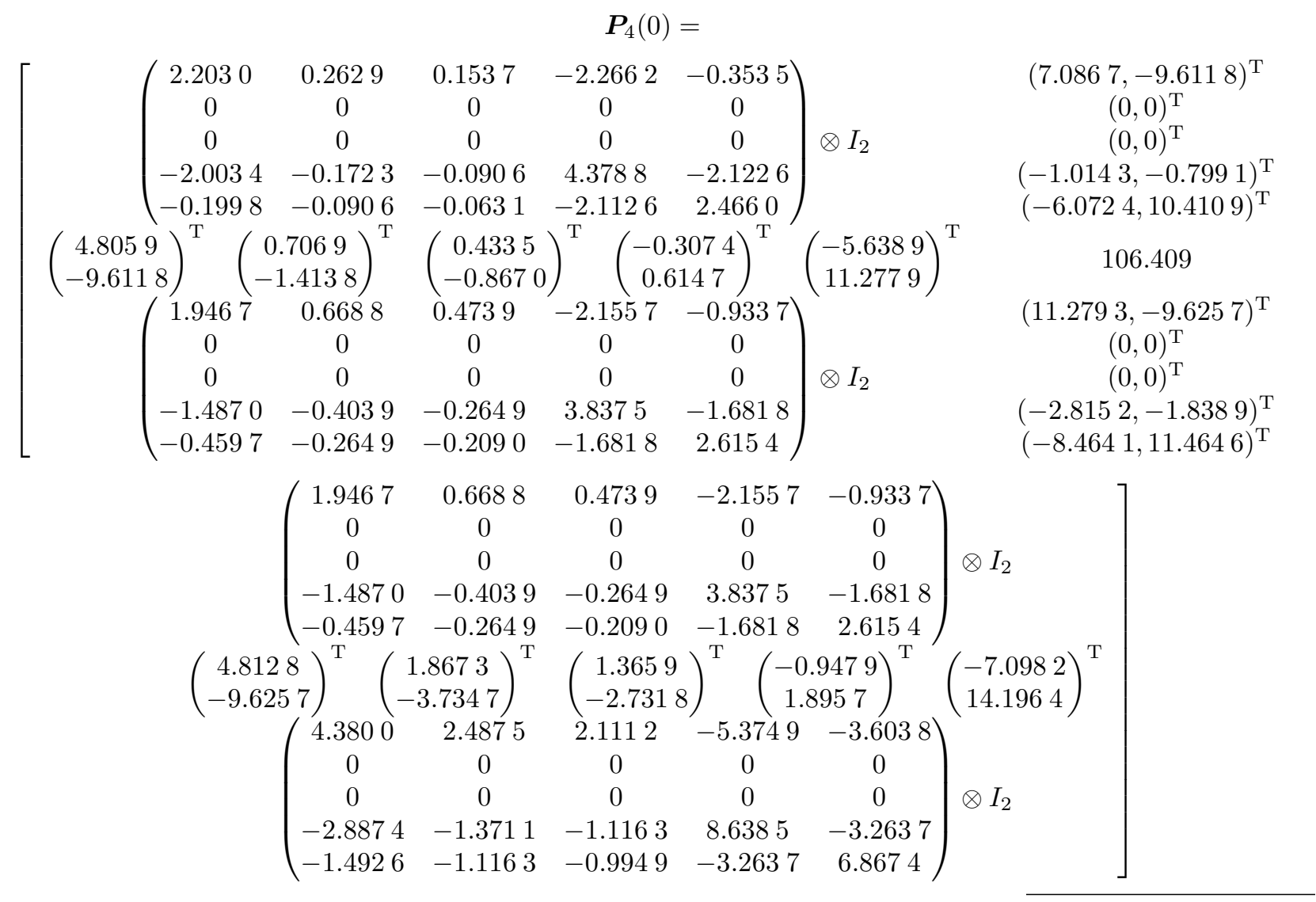

\section{References}

[1] FERREIRA-VAZQUEZ E D, HERNANDEZ-MARTINEZ E G, FLORES-GODOY J J, et al. Distance-based formation control using angular information between robots. Journal of Intelligent and Robotic Systems, 2016, 83(3/4): 543-560.

[2] SAVKIN A V, WANG C, BARANZADEH A, et al. Distributed formation building algorithms for groups of wheeled mobile robots. Robotics and Autonomous Systems, 2016, 75(B): $463-474$

[3] LIU G P, ZHANG S. A survey on formation control of small satellites. Proc. of the IEEE, 2018, 106(3): 440-457.

[4] FU M, YU L, TUO Y. Extended state observer-based distributed formation control for autonomous surface vessels with uncertain disturbances. International Journal of Robotics and Automation, 2018, DOI: 10.2316/Journal.206.2018.1.2064953.

[5] XIE W, MA B, FERNANDO T, et al. A new formation control of multiple underactuated surface vessels. International Journal of Control, 2018, 91(5): 1011-1022.

[6] HAN T, CHI M, GUAN Z, et al. Distributed three-dimensional formation containment control of multiple unmanned aerial vehicle systems. Asian Journal of Control, 2017, 19(4): 1-11.

[7] DONG X, ZHOU Y, REN Z, et al. Time-varying formation control for unmanned aerial vehicles with switching interaction topologies. Control Engineering Practice, 2016, 46: $26-$ 36.

[8] BALCH T, ARKIN R. Behavior-based formation control for multirobot systems. IEEE Trans. on Robotics and Automation, 1998, 14(2): 926 - 939.

[9] ABBASPOUR A, MOOSAVIAN S A A, ALIPOUR K. For- mation control and obstacle avoidance of cooperative wheeled mobile robots. International Journal of Robotics and Automation, 2015, DOI: 10.2316/Journal.206.2015.5.206-4109.

[10] SIRA-RAMIREZ H, CASTRO-LINARES R, PURIEL-GIL G. An active disturbance rejection approach to leader-follower controlled formation. Asian Journal of Control, 2014, 16(2): $382-395$.

[11] REN W. Consensus strategies for cooperative control of vehicle formations. IET Control Theory \& Application, 2007, 1(2): $505-512$.

[12] GU D. A differential game approach to formation control. IEEE Trans. on Control Systems and Technology, 2008, 16(1): 85-93.

[13] BROEK T, WOUW N, NIJMEIJER H. Formation control of unicycle mobile robots: a virtual structure approach. Proc. of the 48th IEEE Conference on Decision and Control and the 28th Chinese Control Conference, 2009: 8328-8333.

[14] ANDERSON G M. Comparison of optimal control and differential game intercept missile guidance laws. Journal of Guidance, Control, and Dynamics, 1981, 4(2): 109-115.

[15] ZHANG G, HUANG C, ZHANG X, et al. Practical constrained dynamic positioning control for uncertain ship through the minimal learning parameter technique. IET Control Theory \& Application, 2018, DOI: 10.1049/ietcta.2018.5036.

[16] ZHANG G, DENG Y, ZHANG W, et al. Novel DVS guidance and path-following control for underactuated ships in presence of multiple static and moving obstacles. Ocean Engineering, 2018, 170(11): $100-110$.

[17] LIN W. Distributed UAV formation control using differential game approach. Aerospace Science and Technology, 2014, 35: 
$54-62$.

[18] MYLVAGANAM T, ASTOLFI A. A differential game approach to formation control for a team of agents with one leader. Proc. of the American Control Conference, 2015: $1469-1474$.

[19] ENGWERDA J C. LQ dynamic optimization and differential games. Chichester: John Wiley \& Sons, 2005.

[20] FREILING G, JANK G, ABOU-KANDIL H. On global existence of solutions to coupled matrx Riccati equations in closed-loop Nash games. IEEE Trans. on Automatic Control, 1996, 41(2): $264-269$.

[21] LI T Y, GAJIC Z. Lyapunov iterations for solving coupled algebraic Riccati equations of Nash differential games and algebraic Riccati equations of zero-sum games. OLSDER G J. Ed. New trends in dynamic games and applications, Annals of the international society of dynamic games. Boston: Birkhäuser, 1995.

[22] AZEVEDO-PERDICOULIS T P, JANK G. Iterative solution of algebraic matrix Riccati equations in open loop Nash games. International Journal of Robust and Nonlinear Control, 2005, 15(2): $55-62$.

[23] MYlVAGANAM T, ASTOLFI A. Dynamic algorithms for solving coupled algebraic Riccati equations arising in mixed $\mathrm{H} 2 / \mathrm{H} \infty$ control for scalar linear systems. Proc. of the 55th IEEE Conference on Decision and Control, 2016: 652-657.

[24] JÓDAR L. Solving coupled Riccati matrix differential systems. Applied Mathematics Letters, 1991, 4(1): 17-19.

[25] PENG H J, ZHANG S, WU Z G, et al. Precise integration method for solving noncooperative LQ differential game. Mathematical Problems in Engineering, 2013, DOI: 10.1155/2013/713725.

[26] LAUB A J. Matrix analysis for scientists \& engineers. Philadelphia: Society of Industrial and Applied Mathematics, 2004.

[27] NAGURKA M L, YEN V. Development of linear quadratic control laws via control parametrization. International Journal of Systems Science, 1992, 23(2): 2125-2139.

[28] BASAR T. Generalized Riccati equation in dynamic games. BITTANTI S, LAUB A J, WILLEMS J C. Ed. The Ric- cati equation. Communications and control engineering series, Berlin: Springer, 1991.

[29] BERNSTEIN D S. Matrix mathematics theory, facts, and formulas. Princeton: Princeton University Press, 2009.

[30] VARGA R S. Geršgorin and his circles. Berlin: SpringerVerlag, 2004.

\section{Biographies}

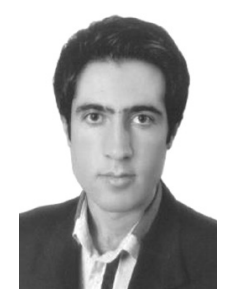

JOND Hossein Barghi was born in 1986. He received his B.S. degree in computer software engineering and M.S. degree in mechatronics engineering from University of Applied Science \& Technology and Islamic Azad University in 2009 and 2011 both from Iran, respectively. Currently, he is working towards his Ph.D. degree in the Department of Computer Engineering, Karadeniz Technical University, Trabzon, Turkey. He spent one year during 2016-2017 in the Department of Applied Mathematics, VSB-Technical University of Ostrava, Czech Republic, within the Erasmus+ exchange program. His research interests include multi-agent systems, optimal control theory, differential game theory, formation control, and trajectory planning. E-mail: barghi@ktu.edu.tr

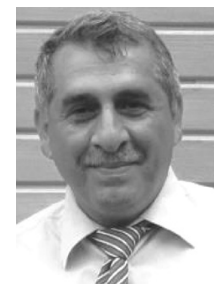

NABIYEV Vasif was born in 1963. He received his B.S. and M.S. degrees in the Faculty of Computer Engineering and Automation from St. Petersburg Electro Technical University in 1985, and his Ph.D. degree in the Department of Computer Science from Moscow Technical University in 1990. From 2005, he has been a professor in Computer Science Department from Karadeniz Technical University, Turkey, where he still lectures. His research interests are in artificial intelligence, biometry, data security, human computer interaction, operational research, discrete and applied mathematics, combinatorial algorithms, and game theory.

E-mail: vasif@ktu.edu.tr 